Music Therapy as an Adjunct in Cardiac Device Lead Extraction Procedures: A randomized controlled trial.

Stine Camilla Blichfeldt-Ærø ${ }^{1}$, Thomas M. Knutsen², Hege Merethe Hagen², Lien My Diep ${ }^{3}$, Gro Trondalen ${ }^{1}$, Sigrun Halvorsen ${ }^{2,4}$

${ }^{1}$ Centre for Research in Music and Health (CREMAH), Norwegian Academy of Music, Slemdalsveien 11, PB 5190, Majorstua, NO-0363 Oslo, Norway

${ }^{2}$ Department of Cardiology, Oslo University Hospital Ulleval, Kirkeveien 166, 0450 Oslo, Norway

${ }^{3}$ Oslo Centre for Biostatistics and Epidemiology (OCBE), Oslo University Hospital, Oslo, Norway

${ }^{4}$ Faculty of Medicine, University of Oslo, Klaus Torgårds vei 3, 0372 Oslo, Norway

Corresponding author:

Stine Camilla Blichfeldt-Ærø, Centre for Research in Music and Health (CREMAH), Norwegian Academy of Music, Slemdalsveien 11, PB 5190, Majorstua, NO-0363 Oslo,

Norway. E-mail address: camillablich@gmail.com. Phone: +47 92498316

\title{
Source of support:
}

The study was a research collaboration between Department of Cardiology at Oslo University Hospital Ulleval and Centre for Research in Music and Health (CREMAH) at the Norwegian Academy of Music.

Number of figures and tables:

4 figures, 3 tables

Conflict of interest

The authors declare no conflict of interest in relation to this work. 


\title{
Music Therapy as an Adjunct in Cardiac Device Lead Extraction Procedures: A randomized controlled trial.
}

\begin{abstract}
:
Background: Evidence of music therapy as an effective supportive therapy in invasive cardiac procedures is increasing, but more research is needed.
\end{abstract}

Aims: To evaluate the impact of music therapy on stress responses during cardiac device lead extraction procedures performed in local anaesthesia.

Methods: Sixty-four patients undergoing cardiac implantable electronic device lead extraction at Oslo University Hospital Ulleval from March 2018 to September 2019 were randomized to music therapy $(n=32)$ or control $(n=32)$. Primary endpoints were patient satisfaction with pain management and average pain intensity during the procedure. Secondary endpoints were average anxiety intensity, need for analgesic/anxiolytic drugs, blood pressure, heart and respiration rate.

Results: All patients in the music therapy group completed the intervention. Patient satisfaction with pain management was $10.00(8.00,10.00)$ in the music therapy vs. 10.00 $(9.00,10.00)$ in the control group $(\mathrm{p}=0.85)$, and average level of pain $0.89(0.22,1.13) \mathrm{vs}$. $0.96(0.36,1.58)$, respectively $(\mathrm{p}=0.38)$. Average anxiety score was $1.00(0.33,2.17)$ in the music therapy vs $1.67(0.71,3.35)$ in the control group $(\mathrm{p}=0.056)$. The use of analgesic/anxiolytic drugs and physiological parameters were similar across groups.

Conclusions. In this study of music therapy during cardiac device lead extractions, no effect was found on patient satisfaction with pain management or average pain level. A decrease in patient anxiety of borderline significance was observed in the music therapy group. More studies with more sensitive measures of pain and anxiety are needed to determine the value of music therapy in invasive cardiac procedures.

Keywords: medical music therapy, procedure support, stress, pain, anxiety, cardiac devices 


\section{Introduction}

The need for extraction of cardiac implantable electronic device (CIED) leads (e. g. pacemakers (PMs) and implantable cardioverter-defibrillators (ICDs) has greatly increased over the past few decades due to the growing demand for device implantations and an accompanying rise in need for extractions due to complications, infections, and lead safety alerts $(1,2)$. Also, increased life expectancy in patients with implanted devices causes increased numbers of device replacements or upgrading procedures to ICD or cardiac resynchronization therapy $(\mathrm{CRT})$ systems $(3,4)$. The number of extraction procedures per year is likely to continue to rise due to the same reasons.

At Oslo University Hospital Ulleval (OUH) in Norway, the extraction procedure is usually performed transvenously in local anaesthesia with some procedural sedation. Analgesic and anxiolytic drugs are given at the start of the invasive procedure and repeated if needed. The invasive phase of the procedure lasts typically between 45 min and 3 hours. Despite the given drugs, most patients will experience some degree of pain and/or anxiety during the procedure; the amount is influenced by the patients' individual ability to cope with stressors, and by extraction techniques and lead dwell time (5).

Music therapy, defined as the use of music and aspects of a therapeutic relationship to promote healthy coping and decrease distress, is increasingly used as procedural support for invasive medical procedures (6-8). As an evidence-based complementary therapy form, music therapy can evoke and regulate mood and emotions (9), reduce distress and anxiety (10-12), and induce changes in heart activity, blood pressure and breathing, in addition to influencing the individual's experience of pain and the surgery itself (13-15).

Several studies on music therapy in patients with heart diseases have been performed, as well as on music listening without a therapist involved $(14,16)$. A systematic review of 26 studies in patients with coronary heart disease found music interventions to decrease distress, 
pain, and anxiety (12). In cardiac catheterization, music therapy was found to decrease preoperative anxiety, especially for patients with high psychological strains (17). A qualitative study found that music therapy might improve the rehabilitation process after cardiothoracic surgery (18). However, the results of studies have been inconsistent, and there is a need for more research on the effect of music therapy in patients with heart disease (12). This is particularly the case in patients undergoing invasive cardiac procedures.

This study aimed to evaluate the possible effects of music therapy used as an adjunct in cardiac device lead extraction procedures performed transvenously in local anaesthesia. We hypothesized that music therapy could decrease pain and anxiety levels during the procedure, and increase patient satisfaction with pain management. The music therapy intervention was tailored for the specific cardiac procedure and provided by a certified music therapist.

\section{Methods}

This study was an open, randomized controlled trial (RCT) performed at a single centre (Department of Cardiology, Oslo University Hospital (OUH) Ulleval, Oslo, Norway). The study was registered in ClinicalTrials.gov no.: NCT04172662.

\section{Cardiac implantable electronic device (CIED) lead extractions}

The PM/ICD centre at the Department of Cardiology, OUH, Oslo, Norway is a high-volume centre for CIED lead extractions, treating patients from all parts of Norway. Approximately 140 lead extractions are performed every year. These procedures are performed transvenously, using a combination of single traction, single sheath mechanical dilatation and femoral and jugular snaring techniques, or other more complicated techniques in selected cases (19). The extraction procedures are performed in a hybrid lab with a cardiothoracic surgery standby-team available but not present, (20-22). Most procedures are performed in local anaesthesia with some procedural sedation; general anaesthesia is used in selected cases (23). All patients are 
prepared with blood pressure and heart rate monitoring and the application of cutaneous pads for defibrillation. The rate of procedural success from 1998 to 2014 was 99\% (19).

The duration of the invasive procedure was defined as the time from the first cut through the skin until the sutures were completed. The total procedure time was defined as the time from arrival at the preoperative room on the day of operation until 2,5 hours postoperatively.

\section{Study population}

All consecutive patients referred to the PM/ICD centre for CIED lead extractions on March 1, 2018, to September 30, 2019, were screened for participation in this study. The inclusion criteria were age 25-80 years, a device implanted $>12$ months ago, planned lead extraction in local anaesthesia, able to speak and read Norwegian, no significant hearing impairment, and willingness to participate in the study. Exclusion criteria were previous and/or acute psychiatric diagnosis, cognitive or mental deficits or impaired functioning. Eligible patients were asked for participation in the study the day before the procedure after verbal and written information was given. After written, informed consent was obtained, the patients were randomly assigned in a 1:1 ratio to the intervention- or the control group. Consecutively numbered, sealed envelopes containing treatment modality according to random numbers were used. The assignment schedule was based on computer-generated random numbers in varying block sizes, and the researchers were not involved in the process.

[Insert Fig. 1: Consort diagram of study participants]

\section{Intervention strategies}

Control group: Patients were given analgesic and anxiolytic drugs according to the following standard procedure: Standard pain management before and during the procedure included 2 tablets of Paralgin forte (paracetamol $400 \mathrm{mg}$ plus codeine $30 \mathrm{mg}$ ) given orally as prophylactic 
medication before entering the operation theatre. At the start of the invasive procedure, the patient received midazolam (1-1.5 $\mathrm{mg})$ and fentanyl $(25-50 \mathrm{mcg})$ intravenously. New doses might be given during the procedure, based on clinical assessment by the medical team and the level of pain and anxiety expressed by the patient.

Music therapy group: The patients randomized to music therapy received the music therapy intervention in addition to the standard drug treatment described above. The music therapy intervention, called "facilitated music listening", was provided by a certified music therapist and has been described in detail previously (24). The protocol was based on biopsychosocial principles of stress- and pain management $(5,25)$, and on receptive music therapy as procedure support (6-8). Briefly, the intervention included a preparatory session the day before the cardiac procedure, sequences of music listening during the pre- and perioperative phase (>30 min.), and the opportunity of music listening the first postoperative period (4 hours). The intervention ended with a closing dialogue between the patient and the music therapist. In the preparatory session, assessment of expectations and music preferences were made, and individual guidance of relaxing strategies for coping with stress. From a set of 12 playlists provided on an iPad application ( "The Music Star") (26), the patient selected 1-3 preferred playlists. These playlists were professionally developed for supportive therapeutic purposes and contained music of different genres and complexity (26). A loudspeaker designed for hospital environments was used, to allow for the necessary communication perioperatively (27). Facilitated music listening could be continued during the first postoperative hours, according to the patient's preferences (24).

\section{Outcomes}

The primary outcomes of the study were 1) patient satisfaction with pain management during the procedure, and 2) the average self-reported pain intensity during the procedure. Secondary 
outcomes were 1) the average self-reported level of anxiety during the procedure, 2) the total amount of anxiolytic drugs given during the procedure, 3) the total amount of analgesic drugs given during the procedure, and 4) average heart rate, average respiration rate, average oxygen saturation and average arterial blood pressure during the procedure.

\section{Measurements}

The measure of patient satisfaction with pain management during the procedure was obtained postoperatively using a 10-point visual numeric scale which has been used in comparable studies $(13,15,28)$. Number one represented very dissatisfied and 10 very satisfied. The measure was answered in privacy, on a paper questionnaire handed to the patient by the music therapist, and returned in a sealed envelope. After the scoring of patient satisfaction, patients in the intervention group were invited to write a brief reflection on their experience with the music therapy intervention.

Patients' self-reported levels of pain and anxiety were assessed using the Numeric Rating Scale (NRS) and the Numeric Visual Analog Anxiety Scale (NVAAS), respectively, with scores from zero to 10 . Zero represented no pain/anxiety, and 10 the worst possible pain/anxiety. The scales are found to be reliable and valid tools, correlating significantly with other measurement tools for pain and anxiety $(29,30)$. The patients were asked to score their level of pain and anxiety before start of the procedure (pre), every 30 min during the procedure (peri), and after the invasive procedure was finished (post). The scores were collected by a nurse from the medical team. The nurses also measured the physiological parameters and registered the use of anxiolytic (diazepam, midazolam) and analgesic drugs (paracetamol/codeine, fentanyl) throughout the procedure. All preoperative measures were obtained before the first dose of midazolam was given.

The patients received verbal and visual instructive information of the numeric rating scales in use and informed about the repeated collection of self-reported measures during the 
procedure. The native language of all participants (Norwegian) was used in all written material and verbal dialogues. The use of central terms in Norwegian (e.g. pain, anxiety, satisfaction) were discussed and internally validated by the research group and medical team. Translation of qualitative data (i.e. written patient reflections) from Norwegian into English was performed by the first author after the analysis. The accuracy of the translation was validated by two researchers independently. These researchers were not involved in the study at any point.

\section{Ethics}

The study was performed in accordance with the guidelines of Good Clinical Practice and CONSORT (31), and Code of Ethics for Music Therapists in Norway. Approval was obtained from the Regional Ethics Committee in Norway (document 2018/168/REK nord). All data were treated and stored de-identified in accordance with national directives.

\section{Statistical analysis}

The power analysis was based on the patient's satisfaction with pain management as the primary outcome. Based on a comparable study, the mean score in the control group was assumed to be 6.5, with a standard deviation of 1.5 (28). We calculated that a sample size of 68 patients would allow us to detect a difference between groups of at least $20 \%$, with a power of $80 \%$ and a significance level of $5 \%$.

Continuous variables were expressed as medians with interquartile ranges (IQR), and categorical variables as frequencies with percentages. Differences between groups were assessed by Mann-Whitney U tests. The average of scores during the procedure (pre-, peri-, and postoperative) for pain and anxiety were calculated. For the perioperative score, the average of repeated perioperative measures ( $\approx$ every $30 \mathrm{~min}$.) was used due to varying duration of the extraction procedures. 
The primary analysis was an intention to treat analysis. The significance level was set at 0.05 (two-sided). Effect sizes, classified as small $(\mathrm{r}=0.10)$, medium $(\mathrm{r}=0.30)$ or large $(\mathrm{r}=0.50)$, were calculated for data showing differences between groups with test results $p<0.2(32)$. Sensitivity analyses were performed for the best- and worst-case, with the imputation of data based on the lower/upper limits of the numeric scale instead of multiple imputation methods, as the distributions of psychometric data were highly skewed and included scores of zero. All data were analysed using IBM SPSS version 25 or Stata/SE15.

The written patient reflections were explored using a content analysis method (33).

\section{Results}

Of 178 screened patients, 78 patients were asked to participate and 68 patients were enrolled in the study (Fig. 1). Four patients were secondarily excluded, due to the violation of inclusion criteria. The median age was $66(56,72)$ years and $46(72 \%)$ were men. Patient characteristics at baseline were similar in the two groups (Table 1). 
Table 1: Baseline characteristics

\begin{tabular}{|c|c|c|}
\hline & $\begin{array}{l}\text { Music therapy } \\
\quad(n=32)\end{array}$ & $\begin{array}{c}\text { Control } \\
(n=32)\end{array}$ \\
\hline \multicolumn{3}{|l|}{ Basic } \\
\hline Females & $8(25.0 \%)$ & $10(31.3 \%)$ \\
\hline Age (years) & $64.5(56.0,72.0)$ & $68.0(54.8,71.8)$ \\
\hline Weight (kg) & $82.0(69.0,97.8)$ & $84.5(77.5,93.8)$ \\
\hline Height $(\mathrm{cm})$ & $176.5(170.0,181.5)$ & $179.5(168.5,185.8)$ \\
\hline \multicolumn{3}{|l|}{ Medical history, n (\%) } \\
\hline Hypertension & $7(21.9 \%)$ & $7(21.9 \%)$ \\
\hline Heart failure & $15(46.9 \%)$ & $16(50.0 \%)$ \\
\hline Myocardial infarction & $8(25.0 \%)$ & $8(25.0 \%)$ \\
\hline Kidney failure & $6(18.8 \%)$ & $3(9.4 \%)$ \\
\hline Diabetes & $7(21.9 \%)$ & $3(9.4 \%)$ \\
\hline Smoker & $5(15.6 \%)$ & $5(15.6 \%)$ \\
\hline \multicolumn{3}{|l|}{ Vital sign } \\
\hline $\begin{array}{l}\text { Systolic blood pressure }(\mathrm{mmHg}) \\
\text { Diastolic blood pressure }(\mathrm{mmHg})\end{array}$ & $\begin{array}{c}126.5(112.5,144.5) \\
70.0(63.5,80.8)\end{array}$ & $\begin{array}{c}115.5(106.3,137.5) \\
74(64.0,82.5)\end{array}$ \\
\hline Heart rate (beats/min) & $68.5(60.0,78.0)$ & $65(58.3,74.8)$ \\
\hline Oxygen saturation, \% & $98.0(96.3,99.0)$ & $97(96,0,98.3)$ \\
\hline \multicolumn{3}{|l|}{ Self-reported scores at baseline } \\
\hline Pain (NRS 0-10) & $0.00(0.00,0.00)$ & $0.00(0.00,1.00)$ \\
\hline Anxiety (NVAAS 0-10) & $2.00(0.00,3.00)$ & $2.00(1.00,4.00)$ \\
\hline
\end{tabular}

Data shown are numbers (\%) or medians (interquartile range)

NRS= Numeric Rating Scale; NVAAS= Numeric Visual Analog Anxiety Scale

The procedure-related variables are shown in Table 2 . Infection and lead failure were the most common reasons for lead extraction. The median duration of the invasive procedure was 58 min. $(36,81)$ in the music therapy group and $55 \min .(32,89)$ in the control group. The median number of years since cardiac device lead implantation was $7.0(3.0,12.8)$ in the music therapy group, and $4.5(3.0,7.8)$ in the control group $(\mathrm{p}=0.18)$. Sheaths were used in $65.6 \%$ of the procedures, with a median duration of $4.5 \mathrm{~min} .(1.5,7.9)$ and $5.4 \mathrm{~min} .(3.0,9.5)$ respectively 
$(\mathrm{p}=0.37)$. The median duration of music listening in the intervention group was $127 \mathrm{~min} .(109$, 156). Only $8 / 32$ patients ( $25 \%$ ) chose to use music listening in the postoperative phase.

Table 2: Procedure variables

\begin{tabular}{|l|c|c|}
\hline & $\begin{array}{c}\text { Music Therapy } \\
(n=32)\end{array}$ & $\begin{array}{c}\text { Control } \\
(n=32)\end{array}$ \\
\hline $\begin{array}{l}\text { Pacemaker } \\
\text { Implantable cardioverter-defibrillator }\end{array}$ & $\begin{array}{c}18(56,3 \%) \\
14(43,8 \%)\end{array}$ & $\begin{array}{c}20(62,5.1 \%) \\
12(37.5 \%)\end{array}$ \\
\hline Years since lead implantation & $7.0(3.0,12.8)$ & $4.5(3.0,7.8)$ \\
\hline $\begin{array}{l}\text { Reason for extraction: } \\
\text { Infection } \\
\text { Eead loosened } \\
\text { Other }\end{array}$ & $15(46.9 \%)$ & $12(37.5 \%)$ \\
$1(3.1 \%)$ & $15(3.1 \%)$ \\
& $5(15.6 \%)$ & $4(12.5 \%)$ \\
\hline Extraction completed & $30(93.8 \%)$ & $26(81.3 \%)$ \\
\hline Number of leads out & $1.00(1.00,2.00)$ & $2.00(1.00,2.00)$ \\
\hline Number of leads in & $1.00(0.25,2.00)$ & $1.00(0.00,2.00)$ \\
\hline Duration invasive procedure (minutes) & $58(36,81)$ & $55(32,89)$ \\
\hline Duration total procedure (minutes) & $325(294,381)$ & $305(270,354)$ \\
\hline Use of Sheaths & $22(68.8 \%)$ & $20(62.5 \%)$ \\
\hline Use of Sheaths - duration (minutes) & $4.5(1.5,7.9)$ & $5.4(3.0,9.5)$ \\
\hline Adverse events * & $1(3.1 \%)$ & $1(3.1 \%)$ \\
\hline
\end{tabular}

Data shown are numbers (\%) or medians (interquartile range)

* Bradycardia in the music therapy group, ventricular tachycardia in the control group

Patient satisfaction with pain management was $10.00(8.30,10.00)$ in the music therapy group vs. $10.00(9.00,10.00)$ in the control group $(\mathrm{p}=0.85)$ (Fig. 2a). The average pain scores during the procedure were $0.89(0.22,1.13)$ in the music therapy group and $0.96(0.36,1.58)$ in the control group ( $\mathrm{p}=0.38)$ (Fig.2b).

[Insert Fig. 2: Levels of patient satisfaction (a) and average of pain scores (b). Data presented as median with interquartile range $\left(25^{\text {th }}\right.$ percentile, $75^{\text {th }}$ percentile $)$, and 'whiskers' $\left(10^{\text {th }}\right.$ percentile, $90^{\text {th }}$ percentile). Min/max values outside 'whiskers' are marked as outliers.] 
The average scores of anxiety during the procedure are found in Figure 3a were $1.00(0.33$, $2.17)$ in the music therapy group and $1.67(0.71,3.35)$ in the control group, showing a difference of borderline significance $(\mathrm{p}=0.056)$. Sensitivity analyses showed that the imputation of missing perioperative data would alter the findings. Best-case calculation found an average of $0.88(0.21,2.17)$ in the music therapy group compared to $1,67(0.71,3.35)$ in the control group, demonstrating a significant difference $(\mathrm{p}=0.04, \mathrm{r}=0.26)$. The worst-case analysis demonstrated an average of $1.00(0.33,2.17)$ in the music therapy group vs. $1.67(0.71,3.35)$ in the control group $(\mathrm{p}=0.08, \mathrm{r}=0.22)$.

[Insert Fig. 3: Average of anxiety scores (a) and use of anxiolytic/analgetic drugs (b). Data presented as median with interquartile range $\left(25^{\text {th }}\right.$ percentile, $75^{\text {th }}$ percentile $)$, and 'whiskers' (10 ${ }^{\text {th }}$ percentile, $90^{\text {th }}$ percentile). Min/max values outside 'whiskers' are marked as outliers.]

There were no significant differences between groups in the use of midazolam $(p=0.55)$ or fentanyl $(\mathrm{p}=0.72)$ during the procedure (Fig. 3b). Physiological parameters during the procedure were similar in both groups (Table 3). Very few adverse events occurred. 
Exploratory analyses (Fig.4) of separate pain scores during the procedure showed lower levels of postoperative pain in the music therapy group $0.00(0.00,2.00)$ compared to the control group $1.00(0.00,2.00),(\mathrm{p}=0.36)$. Analyses of separate anxiety scores showed a trend toward decreased preoperative levels in the music therapy group $2.00(0.00,3.75)$ compared to the control group $3.00(1.00,5.00)(\mathrm{p}=0.16, \mathrm{r}=0.18)$. Postoperative anxiety scores demonstrated a median of $0.00(0.00,0.00)$ in the music therapy group vs $1.00(0.00,2.00)$ in the control group $(\mathrm{p}<0.001, \mathrm{r}=0.47)$.

[Insert Fig.4: Explorative analyses. Pain (a) and anxiety, (b) levels at separate measure points presented as medians.]

Preliminary analyses of the written reflections suggested that patients in the music therapy group expressed positive responses to the music therapy overall, increasing the experienced procedure coping, wellbeing, and satisfaction.

Table 3: Physiological parameters

\begin{tabular}{|l|c|c|c|}
\hline & Music therapy & Control & p-value \\
\hline Blood pressure (mmHg) & & & \\
$\quad$ systolic & $121.50(109.25,139.25)$ & $119.83(103.08,131.33)$ & 0.37 \\
$\quad$ diastolic & $70.00(63.42,80.92)$ & $70.00(60.42,75.92)$ & 0.84 \\
Respiration rate (breaths/min) & $14.33(12.67,15.83)$ & $13.83(12.67,15.58)$ & 0.98 \\
Heart rate (beats/min) & $66.67(58.00,71.83)$ & $64.17(59.08,72.75)$ & 0.93 \\
Oxygen saturation, \% & $96.33(95.33,97.58)$ & $96.00(95.33,96.92)$ & 0.49 \\
\hline
\end{tabular}

Average of scores presented as medians (interquartile range). 


\section{Discussion}

The main goal of this study was to investigate music therapy, in the form of facilitated music listening, as a possible adjunct in regulating stress responses for patients during a cardiac invasive procedure performed in local anaesthesia. The cardiac procedure was transvenous cardiac device lead extraction, an invasive procedure typically entailing some stress and pain for the patient. We measured levels of patient satisfaction, self-reported pain and anxiety, vital signs and procedure medication. Patient satisfaction with pain management was high, and pain levels generally low, in both the music therapy and the control groups. No significant effect was found of music therapy related to pain. For the secondary outcome anxiety, we found a decrease of borderline significance in favour of the music therapy group. The use of analgesic/anxiolytic drugs and vital signs were similar across groups.

To our knowledge, this is the first study to investigate the efficacy of music therapy as an adjunct in regulating stress responses in patients during cardiac device lead extractions performed in local anaesthesia. Despite the use of local anaesthesia and light sedation, such extractions may be painful and do trigger some anxiety in most patients. Based on some previous studies, we hypothesized that music therapy would increase patient satisfaction with pain management and decrease pain levels during the procedure. However, our study did not give support to this hypothesis. Levels of anxiety seemed to be the only variable affected during the music intervention, being lower in the music therapy group.

The low levels of pain in both study groups (median NRS scores $\leq 1$ ) may be one explanation for the lack of effect of music therapy. With low pain levels in the control group, further reductions are hard to achieve. In our study, music therapy was provided on top of the standard procedure, which included moderate doses of analgetic and anxiolytic drugs. A more restrictive administration of analgetic and anxiolytic drugs might have changed the results. The 
pain levels might also be influenced by the fact that the cardiologists performing the lead extractions were very well trained and experienced (19), a factor known to reduce pain levels and the risk of complications during the procedure. Other variables known to influence pain levels during lead extractions, as the number of years since device lead implantation and use of sheaths, were similar across groups (19).

The average anxiety levels measured by NVAAS were lower in the music therapy compared to the control group, but the reduction did not reach statistical significance. In the exploratory analysis, we found reduced anxiety levels in the music therapy group, particularly in the preoperative and postoperative phases. In those phases, the patients were not given midazolam (anxiolytic). In previous research, measures of pain and anxiety have often been limited to either the pre- and postoperative, or solely the postoperative phase $(10,12,15)$. In our study, it was of main importance to include perioperative measures. However, this inclusion might have affected the final results showing less reduction in levels of anxiety than what has been found in previous studies $(10,11,15)$. As the reduction in average level of anxiety was associated with some uncertainty, more research is needed to explore this further.

Although there is previous evidence of music interventions' effects on heart rate, blood pressure and respiration rate, the findings are mixed and often associated with uncertainty (14, $15,34)$. As music interventions may elicit various reactions depending on a merged complexity of contextual, psychosocial and physiological factors, interpreting results of music interventions on physiologic parameters is complex. In our study, the music therapy intervention did not have any effect on physiologic parameters, and further analyses were not made.

Previous studies have shown promising results with music interventions in patients with heart disease $(12,17)$. Our results are in line with studies by Ripley et al. (35) and Palmer et al (36), who found high patient satisfaction overall, but no differences between groups. In contrast, 
Al-Azawy et al. found significantly higher satisfaction in patients who received instructive preparative information, focusing on procedure expectations and coping, combined with premedication (28). It is also in contrast to a previous meta-analysis by Hole et al. on patient satisfaction with care (15), including 16 studies of varied music interventions with patients undergoing surgical procedures.

Previous research reported that music may have pain-reducing effects in patients with coronary heart disease (12) and in surgery $(10,34)$. We did not find such pain-reducing effect in our study. Still, through the exploratory analysis, a small trend was seen towards decreased postoperative pain in the music therapy group, in accordance with the meta-analyses by Kühlmann (10) and Hole (15), evaluating music interventions for surgery patients.

We found that facilitated music listening was applicable as an adjunct during the cardiac invasive procedure, with only one adverse event not related to music therapy. The patients were easily recruited and preferred prolonged music listening far beyond the required minimum of 30 min. The study results indicated that the patients were satisfied with the intervention and had low levels of pain. The patients' general satisfaction and written comments also suggested that music therapy may be desirable among the patients undergoing cardiac device lead extractions, despite the lack of impact on most effect variables in this study.

\section{Limitations}

As in most music therapy studies, the study could not be blinded, implicating a risk of bias. Despite the standardization of both information and measurements, we cannot exclude that differences occurred due to the open design.

Patient satisfaction in this study was higher than expected. Accordingly, the power analysis was based on false estimates. However, with such a high degree of patient satisfaction and low pain levels in the control group, it is unlikely that a higher number of patients would have changed the results of the primary outcome. 
The skewness of the distribution of scores in both groups is a weakness of the measurement tools and the choice of research methodology. The measurement tools were chosen in order to minimize both patient strain and operator distraction during the complex cardiac invasive procedure. However, we cannot exclude that more sensitive tools for measurement of stress responses might have given a different result. Further, the intention of the summary measure might not have been clearly communicated hence perceived by the patients.

As facilitated music listening was defined as an indivisible unit of individual guiding and music listening, no distinction was made between possible effects of the therapeutic relation and the effect of the music itself. A broader scope of patient experiences and interdisciplinary factors should be included in future research designs to better understand the effects of music therapy approaches on various outcomes during invasive cardiac procedures.

\section{Conclusion}

The present study is the first RCT to report outcomes of facilitated music listening as an adjunct in cardiac device lead extraction procedures. The music therapy interventions during the procedures were feasible and safe. Patient satisfaction with pain management was high, and average pain levels low, with no significant differences between groups. A decrease in patient anxiety of borderline significance was observed in the music therapy group. The findings have elements of uncertainty and must be interpreted with caution, and further research is needed.

\section{Acknowledgments}

The study is a research collaboration between the Department of Cardiology at Oslo University Hospital Ulleval, and Centre for Research in Music and Health (CREMAH) at the Norwegian Academy of Music. Conflict of interest: none declared. 


\section{References}

1. Bongiorni M, Di Cori AS, Zucchelli GV, Paperini LM, Coluccia GS. Where is the future of cardiac lead extraction heading? Expert Review of Cardiovascular Therapy. 2016;29 (Aug.):1-7.

2. Wazni O, Wilkoff B. Considerations for cardiac device lead extraction. Nature Reviews Cardiology. 2016;April;13(4):221-9.

3. Deshmukh A, Patel N, Noseworthy P, Patel A, Arora S, Kapa S, et al. Trends in use and adverse outcomes associated with transvenous lead removal in the United States. Circulation. 2015;132(25):2363-71.

4. Sridhar A, Lavu M, Yarlagadda V, Reddy M, Gunda S, Afzal R, et al. Cardiac implantable electronic device-related infection and extraction trends in the U.S. Pacing Clin Electrophysiol. 2017;40(3):286-93.

5. Dornelas EA. Stress proof the heart. Behavioral interventions for cardiac patients. New York NY: Springer; 2012. 408 p.

6. Ghetti CM. Music therapy as procedural support for invasive medical procedures: Toward the development of music therapy theory. Nordic Journal of Music Therapy. 2012;2 (1):3-35.

7. Allen J. Pain management with adults. In: allen JL, editor. Guidelines for music therapy practice in adult medical care. Gilsum: Barcelona Publishers.; 2013. p. 35-61.

8. Heiderscheit A. Surgical and procedural support for adults. In: Allen J, editor. Guidelines for music therapy practice in adult medical care. Gilsum: Barcelona Publishers; 2013. p. 17-34.

9. Grocke D. Receptive music therapy. In: Edwards J, editor. The Oxford handbook of music therapy. Oxford: Oxford University Press; 2016. p. 684-706.

10. Kühlmann AYR, de Rooij A, Kroese LF, van Dijk M, Hunink MGM, Jeekel J. Metaanalysis evaluating music interventions for anxiety and pain in surgery. British Journal of Surgery. 2018; 105:773-83.

11. Bradt J, Dileo C, Shim M. Music interventions for preoperative anxiety. The Cochrane database of systematic reviews. 2013(6):Cd006908.

12. Bradt J, Dileo C, Potvin N. Music for stress and anxiety reduction in coronary heart disease patients. Cochrane Database of Systemic Reviews. 2013(12):Cd006577.

13. Graff V, Cai L, Badiola I, Elkassabany NM. Music versus midazolam during preoperative nerve block placements: a prospective randomized controlled study. Reg Anesth Pain Med. 2019;44:796-9.

14. Koelsch S, Jancke L. Music and the heart. European heart journal. 2015;36(44):30439.

15. Hole J, Hirsch M, Ball E, Meads C. Music as an aid for postoperative recovery in adults: a systematic review and meta-analysis. The Lancet. 2015;386(10004):1659-71.

16. Hanser SB. Music therapy in cardiac health care: current issues in research. Cardiology in review. 2014;22(1):37-42.

17. Argstatter H, Haberbosch W, Bolay HV. Study of the effectiveness of musical stimulation during intracardiac catheterization. Clinical Research in Cardiology. 2006;95(10):514-22.

18. Short A, Gibb H, Fildes J, Holmes C. Exploring the role of music therapy in cardiac rehabilitation after cardiothoracic surgery. A qualitative study using the Bonny method of Guided Imagery and Music. Journal of Cardiovascular Nursing. 2013;28(6):74-81.

19. Knutsen TM, Vanberg P, Steen T, Skretteberg PT, Kamal Z, Platou ES. Single sheath lead extraction, a single center experience of more than 1800 lead extractions. EHRA-2015. 
Europace Abstracts Supplement ( 2015 ) 17 ( Supplement 3 ), iii160: European Society of Cardiology; 2015.

20. Kusumoto FM, Schoenfeld MH, Wilkoff BL, Berul CI, Birgersdotter-Green UM, Carrillo R, et al. HRS expert consensus statement on cardiovascular implantable electronic device lead management and extraction. Heart Rhythm. 2017;14(12):e503-e48.

21. Deharo J, Bongiorni M, Rozkovec A, Bracke F, Defaye P, Fernandez-Lozano I, et al. Pathways for training and accreditation for transvenous lead extraction: a European Heart Rhythm Association position paper. Europace. 2012;14(1) (Jan):124-34.

22. Bongiorni M, Soldati E, Zucchelli G, Di Cori A, Segreti L, De Lucia R, et al. Transvenous removal of pacing and implantable cardiac defibrillating leads using single sheath mechanical dilatation and multiple venous approaches: high success rate and safety in more than 2000 leads. Eur Heart J. 2008;29(23):2886-93.

23. Wilkoff BL. Transvenous lead extraction: Heart Rhythm Society expert consensus on facilities, training, indications, and patient management. Heart rhythm. 2009;6:1085-104. 24. Blichfeldt-Ærø SC, Trondalen G, Halvorsen S. Facilitated music listening: Music therapy in an invasive cardiac procedure. British Journal of Music Therapy. 2019;33(1):2738.

25. Melzack R. Pain and the neuromatrix in the brain. Journal of Dental Education. 2001;65(12):1378-82.

26. Lund HN, Bertelsen LR, Bonde LO. Sound and music interventions in psychiatry at Aalborg University Hospital. SoundEffect. 2016;(1):48-68.

27. AudioCura. AudioCura M2 AudioCura.com: AudioCura - Music intervention for health care; 2016 [Available from: http://www.audiocura.com/da/portfolio/audiocura-m2/. . 28. Al-Azawy M, Oterhals K, Fridlund B, Assmus J, Schunster P. Premedication and preoperative information reduces pain intensity and increases satisfaction in patients undergoing ablation for atrial fibrillation: a randomised controlled study. Applied Nursing Research. 2015;28:268-73.

29. Hawker GA, Mian S, Kendzerska T, French M. Measures of adult pain. Arthritis Care \& Research. 2011;63(S11):240-52.

30. Elkins G, Staniunas R, Rajab MH, Marcus J, Snyder T. Use of a numeric visual analog anxiety scale among patients undergoing colorectal surgery. Clinical Nursing Research. 2004;13(3):237-44.

31. Boutron I, Altman DG, Moher D, Schulz KF, Ravaud P. CONSORT Statement for randomized trials of nonpharmacologic treatments: A 2017 update and a CONSORT extension for nonpharmacologic trial abstracts. Annals of Internal Medicine.167(1):40-7. 32. Pallant J. SPSS survival manual. 6 ed. Berkshire, England: Open University Press; 2016.

33. Krippendorff K. Content analysis. An introduction to its methodology. 4th ed. California: Sage Publications Inc; 2019.

34. Nilsson U. The anxiety- and pain-reducing effects of music interventions: a systematic review. AORN Journal. 2008;87(4):780-807.

35. Ripley L, Christopoulos G, Michael TT, Alomar M, Rangan BV, Roesle M, et al. Randomized controlled trial on the impact of music therapy during cardiac catheterization on reactive hyperemia index and patient satisfaction: the Functional Change in Endothelium After Cardiac Catheterization, With and Without Music Therapy (FEAT) study. The Journal of invasive cardiology. 2014;26(9):437-42.

36. Palmer JB, Lane D, Mayo D. Surgical music therapy: The significance and implementation of music therapy in the operating arena. Music Therapy Perspectives. 2017;35(1):30-5. 


\section{Figure Legends}

Figure 1. Consort diagram of study participants.

Figure 2. Levels of patient satisfaction (a) and average of pain scores (b). Data presented as median with interquartile range (25th percentile, 75th percentile), and 'whiskers' (10th percentile, 90th percentile). Min/max values outside 'whiskers' are marked as outliers.

Figure 3. Average of anxiety scores (a) and use of anxiolytic/analgetic drugs (b). Data presented as median with interquartile range (25th percentile, 75th percentile) and 'whiskers' (10th percentile, 90th percentile). Min/max values outside 'whiskers' are marked as outliers.

\section{Figure 4.}

Explorative analyses. Pain (a) and anxiety (b) levels at separate measure points presented as medians.

*significant difference 
Fig.1

\section{CONSORT DIAGRAM}






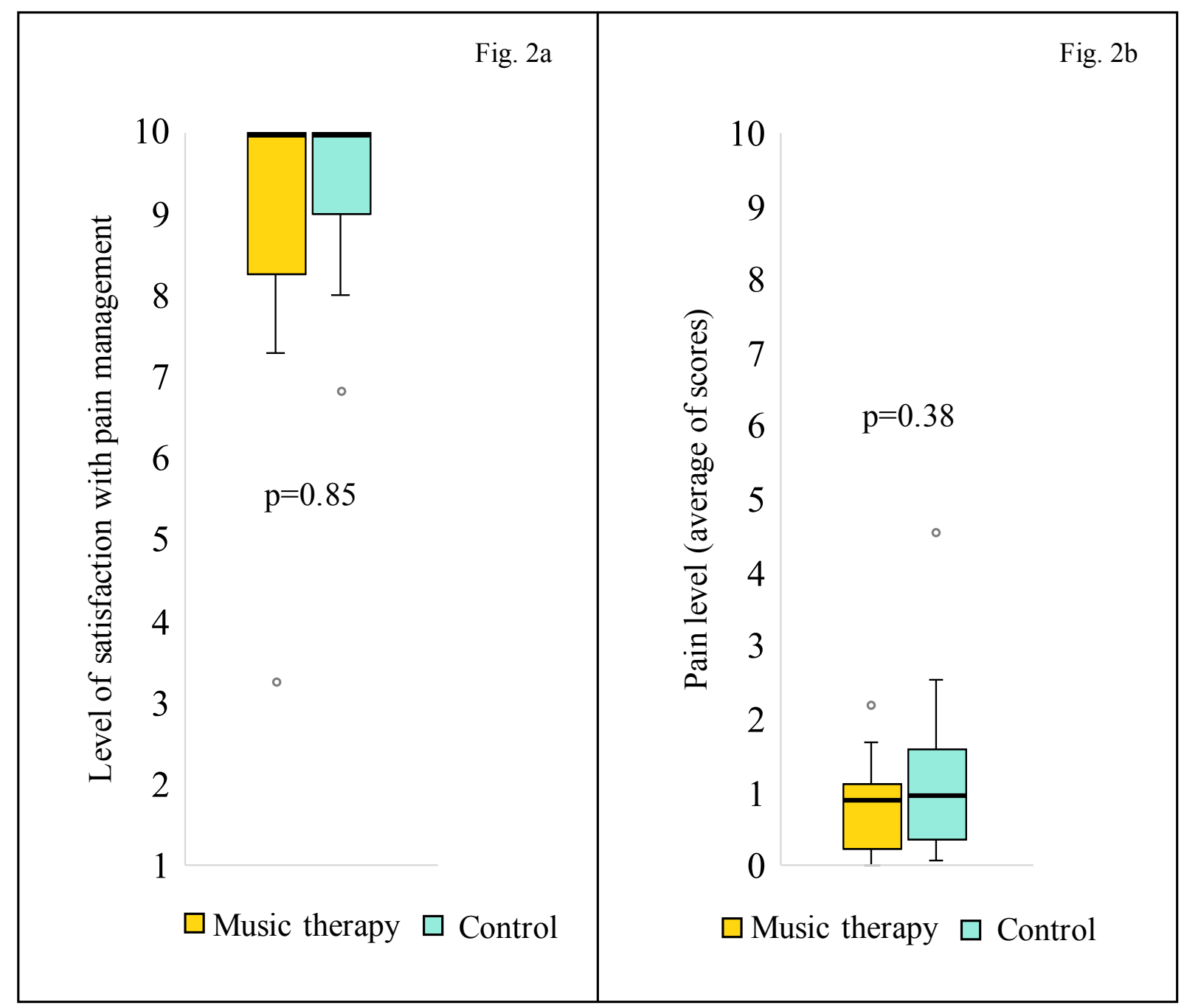




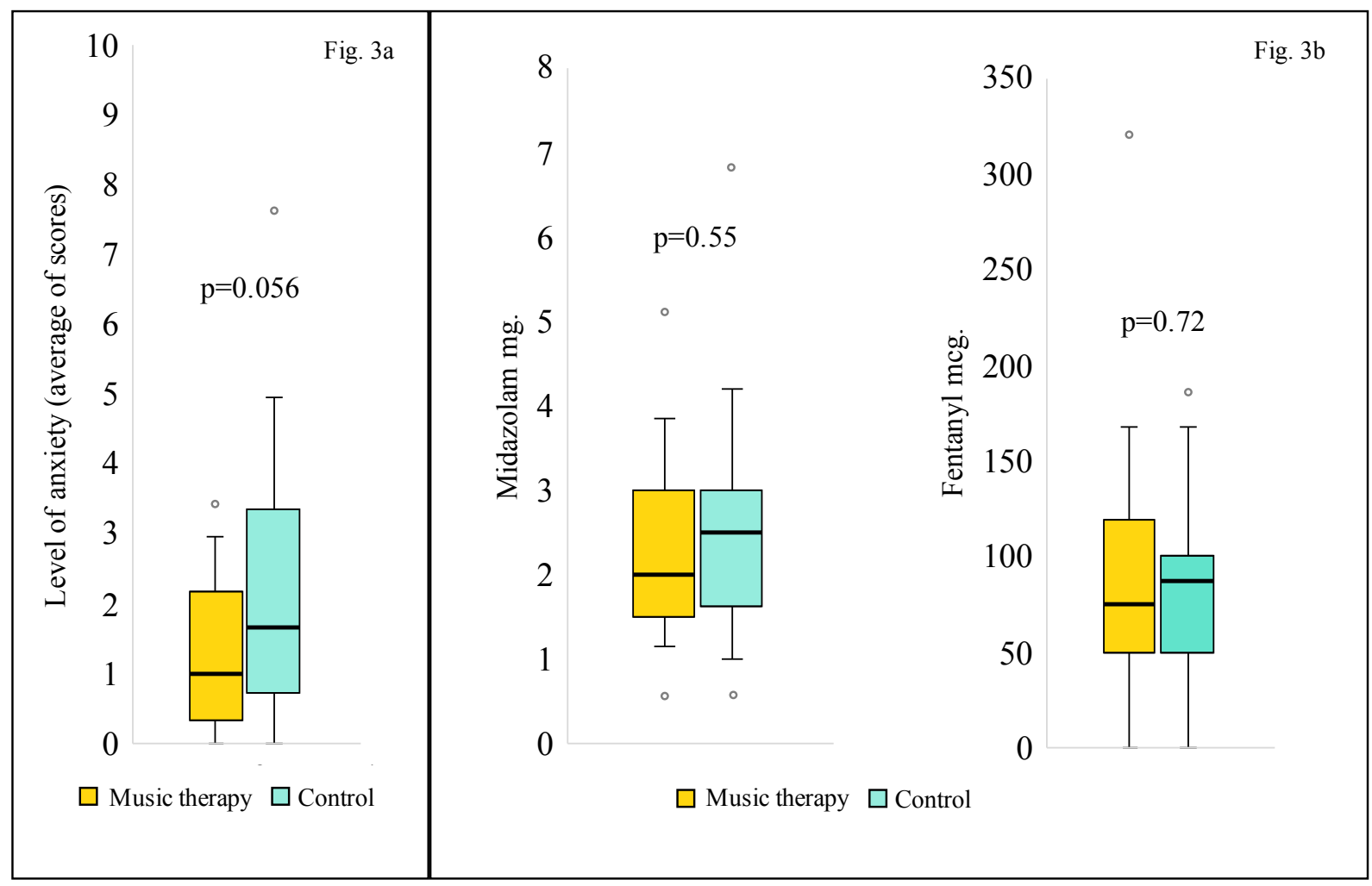




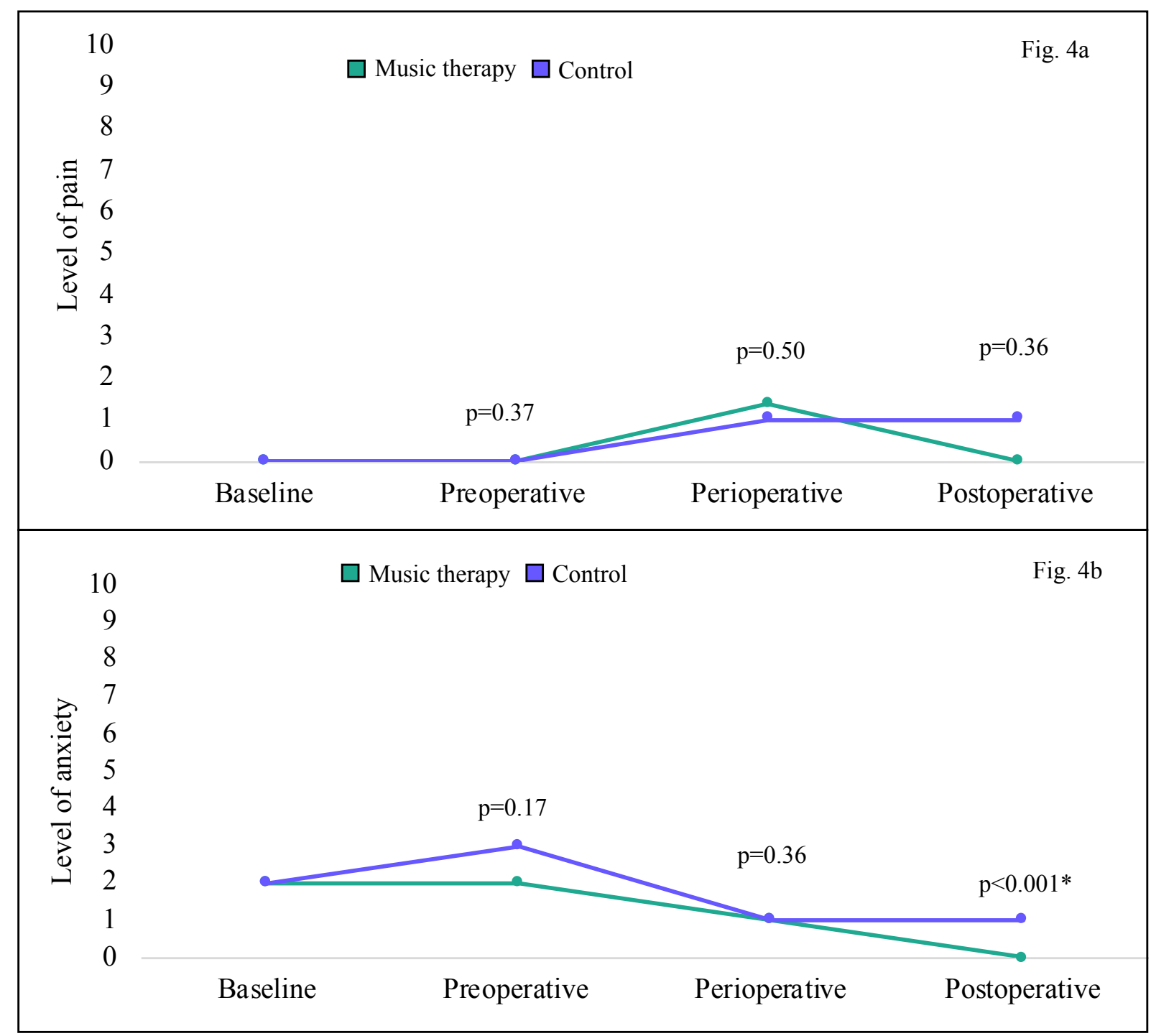




\section{Author statement}

All persons who meet authorship criteria are listed as authors, and all authors certify that they have participated sufficiently in the work to take public responsibility for the content, including participation in the concept, design, analysis, writing, or revision of the manuscript. Furthermore, all authors certify that this material has not been and will not be published in any other journal before its appearance in Applied Nursing Research.

\section{Authorship contributions}

S.C.B.-AE., G.T. and S.H. conceived and planned the project; S.C.B.-AE T.M.K. and H.M.H. performed the experiment and contributed to acquisition of data; S.C.B.-AE and L.M.D. contributed with data analysis and interpretation, with help from S.H; S.C.B.-AE., G.T. and S.H were writing and revising the manuscript, with small contributions from all authors. 